\title{
Modified Overall Immune Related Response Criterion, Partial Response
}

National Cancer Institute

\section{Source}

National Cancer Institute. Modified Overall Immune Related Response Criterion, Partial

Response. NCl Thesaurus. Code C111085.

The evaluation of the antitumor response shows that the sum of the longest diameters of target and new measurable lesions has decreased by at least 30\%. 